\title{
COVID-19 Prevention and Protecting Sex Workers: A Call to Action
}

\author{
Randi Singer ${ }^{1}\left[\right.$ Datasha Crooks $^{2} \cdot$ Amy K. Johnson $^{3} \cdot$ Alexandra Lutnick $^{4} \cdot$ Alicia Matthews $^{5}$
}

Received: 26 August 2020 / Revised: 17 September 2020 / Accepted: 22 September 2020 / Published online: 14 October 2020

(c) Springer Science+Business Media, LLC, part of Springer Nature 2020

The COVID-19 pandemic has disproportionately impacted people of color as well as those affected by systemic poverty (Gross et al., 2020; Human Rights Campaign, 2020). Urbanbased sex workers are another subpopulation at increased risk for contracting COVID-19. Unstable housing, IV drug use, increased criminalization, and limited access to care put sex workers at greater risk of contracting COVID-19 (Human Rights Campaign, 2020; Platt et al., 2020) Although the term sex worker can be defined in multiple ways, for the purpose of this Letter, sex workers are adults who trade oral, anal, and/or vaginal sex for something of value. Although largely neglected in current COVID-19 risk reduction efforts, it is well established that the health and well-being of sex workers is inextricably linked to the health and wellbeing of their clients and to the broader public (Patterson et al., 2009). As such, tailored harm reduction interventions aimed at increasing the use of personal protective equipment among sex workers and their clients may help to reduce the overall burden of COVID-19 infections, hospitalizations, and resulting deaths in urban settings.

The social response, isolation, experience of stigma and the health disparities of COVID-19 are, in many ways, comparable to that of the HIV/AIDS pandemic, especially as it applies to those engaged in sex work. Similar to HIV,

Randi Singer

rbsinger@uic.edu

1 Department of Human Development Nursing Science, College of Nursing, University of Illinois Chicago, $845 \mathrm{~S}$. Damen Ave., Chicago, IL 60612, USA

2 Department of Human Development Nursing Science, College of Nursing, University of Illinois Chicago, Chicago, IL, USA

3 Feinberg School of Medicine, Northwestern University, Chicago, IL, USA

4 Aviva Consulting, University of California, Berkeley, CA, USA

5 Department of Population Health Nursing Science, College of Nursing, University of Illinois Chicago, Chicago, IL, USA complex structural and social factors create unique challenges to targeting COVID-19 health promotion and disease prevention efforts for sex workers (Shannon et al., 2018). Those who exchange sex may regularly experience the social repercussions of stigmatized and criminalized work (Benoit, Jansson, Smith, \& Flagg, 2018; Lutnick, 2019), including a lack of access to health care (Krüsi et al., 2014; Shannon et al., 2018). Research has shown that some sex workers also report increased experiences of homelessness, unemployment, incarceration, mental illness, drug use, and various forms of personal and structural/systemic violence and abuse (Benoit et al., 2019; Human Rights Campaign, 2020; Krüsi et al., 2014; Platt et al., 2020). All of these intersecting experiences impact sex workers' ability to access health care and limit their ability to adhere to COVID-19 prevention practices. Furthermore, criminalization, targeted policing, and environments that limit worker agency impact sex workers' abilities to practice harm reduction and health promotion behaviors (Benoit et al., 2019; Herek 2004; Shahmanesh, Patel, Mabey, \& Cowan, 2008; World Health Organization 2014). As with successful HIV prevention initiatives with sex workers, targeted COVID-19 prevention efforts must also take into account structural barriers to virus prevention and health promotion.

Sex workers would benefit from COVID-19 interventions informed by evidence-based, community-empowered HIV prevention initiatives. For example, Getting to Zero (GTZ) new HIV infections by 2030 is an important public health plan that recognizes the significance of increasing HIV prevention, and the role of self-management and harm reduction for marginalized populations. Similar to community-empowered HIV responses, targeted COVID-19 prevention efforts are needed for people engaged in sex work (World Health Organization et al., 2013).

In this Letter, we draw on preliminary analysis from our study, Centering and Empowerment: The Road to Reducing HIV and STIs in Chicago's Sex Worker Community, to inform public health researchers, clinicians, and health educators about the current status of sex work during the 
pandemic. ${ }^{1}$ As part of the study, funded by the Rita and Alex Hillman Foundation, we conducted semi-structured qualitative interviews with 21 people engaged in sex work to learn about their health needs and experiences. Of the sex workers interviewed, 52\% $(n=11)$ identified as either Black, Latinx, American Indian or Asian American, with 48\% $(n=10)$ identifying as white. A total of $52 \%(n=11)$ identified as cisgender women, $33 \%(n=7)$ identified as transgender or gender fluid, $10 \%(n=2)$ identified as cisgender men, and $5 \%(n=1)$ declined to answer. The majority $(81 \%, n=17)$ identified as either queer, bisexual, pansexual, or gay, $14 \%$ $(n=3)$ participants identified as heterosexual and 5\% $(n=1)$ identified as asexual. Interviews were completed during Illinois' strict first phase of shelter-in-place where schools and all non-essential businesses were closed due to COVID-19. This diverse sample of participants described the barriers to accessing health care they have experienced during and prior to the pandemic. These interviews shed light on the fact that, even during state level shelter-in-place, sex workers needed to and did continue to work. Even though 52\% $(n=11)$ of participants interviewed reported continuation of in-person sex work, nearly all of the interview respondents $(n=19)$ reported a decrease in their earnings due to the pandemic.

This decrease in earnings is due to a decrease in regular clientele. With trusted regular clients no longer available, participants reported that the need for money can sometimes outweigh an ability to vet new clients, making it difficult for some sex workers to feel safe from COVID-19. One participant stated "Well, now that COVID is here, all of my regulars have vanished, so I have been forced to see a lot of new people, potentially exposing me." Although most participants have continued in-person sex work, most had not been tested for COVID-19 due to concern for expense. Some reported wearing personal protective equipment (PPE) in transit to and from client visits; however, nobody acknowledged wearing PPE while doing sex work. Participants were concerned about the inherent risk of seeing clients but were also quick to say that they would isolate for 14 days if they contracted COVID-19. One participant stated "I am concerned that, you know, every time I go out and see a client, I don't know where they've been. I don't know what they've been doing and they could lie to me." When asked about their risk for contracting COVID-19, some sex workers acknowledged that their work puts them at greater risk of contracting COVID-19. As one person shared, "I feel like I' $m$ taking a risk now and that scares me a little bit because it's like, you know, do I need this money that bad." Based on these emerging findings, we conclude that in order to prevent COVID-19 among sex workers, PPE, COVID-19 testing, and information/educational resources related to engaging in sex during COVID-19 must be free, accessible, and widely available.

Working with highly stigmatized populations during public health pandemics is not new. Past HIV public health campaigns utilizing a no questions asked platform have been successful at increasing condom use and reducing rates of HIV and STIs. For example, in 2006, the New York City Department of Health and Mental Hygiene made free condoms available to organizations through a Web-based ordering system. Organizations reported 80\% (280/351) of patrons who saw free condoms, took free condoms, and $73 \%$ (205/280) of those who reported taking them also reported using them (Renaud et al., 2009). In 2019, a systematic literature review of peer-reviewed articles published between 1990 and 2017 including 29 articles from 6 countries looked at condom availability programs (CAPs) (Algur, Wang, Friedman, \& Deperthes, 2019). The majority of studies showed an increase in condom uptake and use at last intercourse. CAPs also correlated with a decrease in rates of STIs. Expanding such programs to include PPE and pamphlets related to sexual safety in the time of COVID-19 would not only benefit the health and well-being of those engaged in sex work, but could also extend to the sexual safety for the general public.

Although various local government and nonprofit agencies in the U.S. have issued guidance about navigating sex during COVID-19, we still do not have guidance from federal agencies like the Centers for Disease Control. The British Columbia Ministry of Health (2020) has issued such guidance and recently published guidelines on how to increase safety and prevention for those who are sexually active, including those engaged in sex work. Their recommendations include, but are not limited to, using masks during intercourse, limiting face-to-face contact, avoiding heavy breathing, and utilizing a "glory hole" (a hole in the wall through which oral, anal, or vaginal penetration occurs without face-to-face contact). The U.S. must follow suit and adapt these guidelines to meet the COVID-19 prevention needs of sex workers and others who are engaging in sex with people who they are not practicing social distancing with (i.e., someone they live with or someone with whom they have had conversations about reducing COVID-19 exposure risks).

This call to action is supported by Howard Brown Health $(\mathrm{HBH})$, a federally qualified health center (FQHC) system in Chicago, Illinois. This FQHC exists to eliminate the disparities in health care experienced by lesbian, gay, bisexual, and transgender people through research, education, and the provision of services that promote health and wellness. In order to encourage people to stay sheltered amidst this pandemic, $\mathrm{HBH}$ has pivoted to meet the changing needs of community members in three ways.

$\overline{1}$ https://healthstudy.uic.edu/ 
1. Delivering at-home free safer sex kits, including water, silicone- and aloe-based lubrication, a variety of condoms, and masks.

2. Delivering at-home free safer injection kits, including sterile water, cotton balls, band aids, and alcohol swabs.

3. Delivering at-home HIV testing kits to patients along with a health educator visit to interpret results and talk through next steps.

Since launching these programs in April 2020, HBH has delivered 5780 condoms, 2356 packages of lubricant, 67 safer injection kits, 128 cloth masks, and 335 HIV home screening kits. HBH is ready to expand this outreach by delivering PPE and educational resources for those engaged in transactional sex.

We have witnessed how slow the pandemic responses have been and they will continue to move at this rate until there is a vaccination available. Therefore, some changes are necessary to ensure the safety of sex workers. At the core of our recommendation is cultural safety (Kellett \& Fitton, 2017). Health promotion and disease prevention must be accessible and safe for all. In order to improve public health outcomes for COVID-19, we must be willing to serve the needs of all people and take into consideration what they do for work and how that work may impact their safety. All people deserve to feel safe when accessing resources for health promotion and disease prevention. A "no questions asked" policy of free and accessible PPE, free and accessible COVID-19 testing, and free and accessible information/educational resources should be available in clinics where free HIV prevention services are offered. Information/educational resources for sex workers need to accurately capture their voices and experiences, be produced promptly, and be accessibly located. More research and resources are needed to protect individuals who engage in sex work during the pandemic, specifically at the intersection of COVID-19 and Black Lives Matter.

\section{References}

Algur, E., Wang, E., Friedman, H. S., \& Deperthes, B. (2019). A systematic global review of condom availability programs in high schools. Journal of Adolescent Health, 64(3), 292-304. https:// doi.org/10.1016/j.jadohealth.2018.11.013.

Benoit, C., Jansson, S. M., Smith, M., \& Flagg, J. (2018). Prostitution stigma and its effect on the working conditions, personal lives, and health of sex workers. Journal of Sex Research, 55(4-5), 457-471. https://doi.org/10.1080/00224499.2017.1393652.

Benoit, C., Maurice, R., Abel, G., Smith, M., Jansson, M., Healey, P., et al. (2019). 'I dodged the stigma bullet': Canadian sex workers' situated responses to occupational stigma. Culture Health and Sexuality, 22, 81-95. https://doi.org/10.1080/13691058.2019.15762 26.

British Columbia Ministry of Health. (2020). COVID-19: Guidance for Sex Workers (Coronavirus COVID-19). BC CDC. https://www.
bccdc.ca/Health-Info-Site/Documents/COVID19_SexWorkers Guidance.pdf

Gross, C. P., Essien, U. R., Pasha, S., Gross, J. R., Wang, S.-Y., \& NunezSmith, M. (2020). Racial and ethnic disparities in population-level Covid-19 mortality. Journal of General Internal Medicine. https ://doi.org/10.1007/s11606-020-06081-w.

Herek, G. M. (2004). Beyond "homophobia": Thinking about sexual prejudice and stigma in the twenty-first century. Sexuality Research and Social Policy, 1(2), 6-24. https://doi.org/10.1525/ srsp.2004.1.2.6.

Human Rights Campaign. (2020). The lives and livelihoods of many in the LGBTQ community are at risk amid COVID-19 crisis (No. COVID19-IssueBrief-032020). https://assets2.hrc.org/files /assets/resources/COVID19-IssueBrief-032020-FINAL.pdf? $\mathrm{ga}=2.245594500 .1637528607 .1596592484-720055857.1596 \overline{5}$ 92484

Kellett, P., \& Fitton, C. (2017). Supporting transvisibility and gender diversity in nursing practice and education: Embracing cultural safety. Nursing Inquiry, 24(1), e12146. https://doi.org/10.1111/ nin. 12146.

Krüsi, A., Pacey, K., Bird, L., Taylor, C., Chettiar, J., Allan, S., et al. (2014). Criminalisation of clients: Reproducing vulnerabilities for violence and poor health among street-based sex workers in Canada-a qualitative study. British Medical Journal Open, 4(6), e005191. https://doi.org/10.1136/bmjopen-2014-005191.

Lutnick, D. A. (2019). The 'prioritizing safety for sex workers policy': A sex worker rights and anti-trafficking initiative. Anti-Trafficking Review, 12, 140-154. https://doi.org/10.14197/atr.201219129.

Patterson, T. L., Goldenberg, S., Gallardo, M., Lozada, R., Semple, S. J., Orozovich, P., et al. (2009). Correlates of HIV, sexually transmitted infections, and associated high-risk behaviors among male clients of female sex workers in Tijuana, Mexico. AIDS, 23(13), 1765-1771. https://doi.org/10.1097/QAD.0b013e32832f08a1.

Platt, L., Elmes, J., Stevenson, L., Holt, V., Rolles, S., \& Stuart, R. (2020). Sex workers must not be forgotten in the COVID-19 response. The Lancet, 396(10243), 9-11. https://doi.org/10.1016/ S0140-6736(20)31033-3.

Renaud, T. C., Bocour, A., Irvine, M. K., Bernstein, K. T., Begier, E. M., Sepkowitz, K. A., et al. (2009). The free condom initiative: Promoting condom availability and use in New York city. Public Health Reports, 124(4), 481-489. https://doi.org/10.1177/00333 5490912400404.

Shahmanesh, M., Patel, V., Mabey, D., \& Cowan, F. (2008). Effectiveness of interventions for the prevention of HIV and other sexually transmitted infections in female sex workers in resource poor setting: A systematic review. Tropical Medicine and International Health, 13(5), 659-679. https://doi.org/10.111 1/j.1365-3156.2008.02040.x.

Shannon, K., Crago, A.-L., Baral, S. D., Bekker, L.-G., Kerrigan, D., \& Decker, M. R. (2018). The global response and unmet actions for HIV and sex workers. The Lancet, 392(10148), 698-710. https:// doi.org/10.1016/S0140-6736(18)31439-9.

World Health Organization. (2014). Consolidated guidelines on HIV prevention, diagnosis, treatment and care for key populations.https ://www.who.int/hiv/pub/guidelines/keypopulations/en/

World Health Organization, United Nations Population Fund, Joint United Nations Programme on HIV/AIDS, Global Network of Sex Work Projects, The World Bank. (2013). Implementing comprehensive HIV/STI programmes with sex workers: Practical approaches from collaborative interventions. Geneva: World Health Organization.

Publisher's Note Springer Nature remains neutral with regard to jurisdictional claims in published maps and institutional affiliations. 